\title{
Flow and pressure characteristics in rectangular channels with internal cylindrical bodies
}

\author{
Hendrik Gossler ${ }^{\mathrm{a}}$, Benjamin L. Kee ${ }^{\mathrm{b}}$, Huayang Zhu ${ }^{\mathrm{b}}$, Matthias Hettel ${ }^{\mathrm{a}}$, Olaf \\ Deutschmann $^{\mathrm{a}}$, Robert J. Kee ${ }^{\mathrm{b}, *}$ \\ ${ }^{a}$ Institute for Chemical Technology and Polymer Chemistry, Karlsruhe Institute of \\ Technology, Karlsruhe, Germany \\ ${ }^{b}$ Department of Mechanical Engineering, Colorado School of Mines, Golden, CO 80401, \\ USA
}

\begin{abstract}
This paper develops models for steady-state, fully developed, laminar flow in rectangular channels with internal coaxial solid cylinders. By casting and solving the parallel-flow momentum equation in a dimensionless setting, correlations are derived for the friction factor $f$ and represented as Re $f$ where Re is the Reynolds number. The correlations consider three positions for the internal cylinder. The cylinder may be in the center of the rectangular channel, in the corner of the channel, or resting at the middle of the channel floor. The correlations incorporate channel aspect ratios in the range $0.1 \leq$ $\alpha \leq 1.0$. The cylinder-diameter aspect ratios $\beta$ range from being vanishingly small to being large enough to touch the channel walls. Although the results are general, the study is motivated by considering the effects of diagnostic probes within small channels of catalytic monoliths.
\end{abstract}

Keywords:

\footnotetext{
*Corresponding author. Tel: (303) 273-3379.

Email address: rjkee@mines.edu (Robert J. Kee)
} 
Catalyst monolith, Capillary probe, Friction factor correlation, Rectangular channels, Pressure drop, Laminar flow

2

3

4

5

\section{Introduction}

Small-channel devices, such as catalyst monoliths (e.g., Fig. 1), are used in a wide range of technologies. Example applications include exhaust-gas after treatment and catalytic partial oxidation of hydrocarbon fuels. Because individual channel cross-sectional dimensions are typically small (i.e., order of millimeters), measuring temperature and composition within individual channels is difficult. A probe-based approach for measuring species composition was first reported by Partridge et al. [1]. Moving the capillary probe along the length of a channel enables the measurement of axially resolved concentration profiles. The approach has been applied and extended by others and also includes temperature measurements with thermocouple probes [1-13].

[Figure 1 about here.]

Considering the small dimensions of the channels in a typical monolith, the presence of physical probes may influence the measurement profiles. Ideally, "capillary" probes should be sufficiently small and positioned so as to only minimally affect the flow through the channels, and thus the interpretation of the measurement. However, even small variations in channel dimensions, and hence the influence of an embedded probe, strongly affect the flow rate and pressure drop within small channels. Results of the present study 
show that placing the small probe in the corner of a relatively larger channel leads to minimal flow-field perturbations. However, larger or centrally positioned probes can cause major flow restrictions.

The modeling approach here is based upon laminar, fully developed, parallel flow within long rectangular channels with an internal solid cylinder. The two-dimensional momentum equation is formulated and solved in a dimensionless framework. The results predict the dimensionless Reynoldsnumber-friction-factor product $\operatorname{Re} f$ as a function of the channel aspect ratio, cylinder diameter, and cylinder placement. Because the model is based upon fully developed flow, the results under-predict the friction factor in developing-flow regions, such as the channel entrance region. Nevertheless, for typically low Reynolds number flow in small channels, the development regions are short. Hettel et al. [9] have developed detailed three-dimensional computational fluid dynamics (CFD) models to evaluate probe effects within catalyst monoliths. These models accurately represent the entry-length development regions, which the present model neglects by assuming fully developed flow. However, the present model provides easily used correlations for a wide range of channel and probe geometries.

Texts on heat exchangers provide friction-factor correlations (i.e., Ref) for numerous channel cross-sectional shapes (cf., Shaw and Sekulić [14]). Most basic fluid mechanics texts give correlations for circular and rectangular channels. However, it is rare to find correlations for channels with internal obstructions. Thus, the present results provide new and general correlations. Section 4 provides illustrative examples and Section 5 discusses how to apply the correlations in plug-flow models. 
The present paper develops the theory and models to evaluate the fluid mechanical influence of axially aligned cylinders within rectangular flow channels. The results provide quantitative tools with which to evaluate the extent of any potential invasive behavior, and thus to assist the engineering design of a probe system. Although certainly motivated by applications such as probes in monoliths, the approach and results are more general. Because of their generality with respect to rectangular-channel dimensions, cylinder diameter, and cylinder placement, the new correlations are potentially useful for other, even unforeseen, applications.

\section{Model development}

The modeling follows a traditional approach of solving a dimensionless axial momentum equation for laminar, fully developed parallel laminar flow in a channel [14-16]. The two-dimensional velocity distribution must satisfy the axial momentum equation as

$$
\nabla^{2} u=\frac{1}{\mu} \frac{\mathrm{d} p}{\mathrm{~d} z}
$$

where $u$ is the velocity, $p$ is the pressure, $\mu$ is the dynamic viscosity, and $z$ is the axial coordinate. Because the flow is fully developed, and assuming constant viscosity, the right-hand side must be a constant. The solution to this elliptic equation requires boundary conditions. Assuming no-slip conditions, the velocity on all the solid boundaries (channel walls and probe surface) vanishes (i.e., $u=0$ ).

To facilitate transformation to a dimensionless setting, the characteristic length scale may be represented in terms of a hydraulic diameter or the square 
root of the cross-sectional area. Solving the partial differential equation yields Re $f$ as a function of the channel geometry. Figure 2 illustrates a rectangular channel that measures $a$ high and $b$ wide. A circular probe of diameter $d$ is placed at the center of the channel. Assume that $a \leq b$ and $d \leq a$. Aspect ratios for the rectangular channel and the cylinder may be defined as $\alpha=a / b$ and $\beta=d / b$, respectively. These aspect ratios are related and bounded as

$$
\beta<\alpha \leq 1, \quad 0<\beta<\alpha
$$

These bounds enforce the constraint that the probe dimensions cannot exceed those of the rectangular channel and that the cylinder cannot vanish entirely (i.e., beta $=0$ ). The case of beta $=0$ (i.e., rectangular channel without a cylinder) is physically valid, leading to well-known solutions. However, the present model formulation imposes a no-slip boundary condition at the cylinder wall, which cannot be imposed in the absence of the cylinder.

[Figure 2 about here.]

\subsection{Characteristic scales}

Generality can be achieved by casting the problem in dimensionless variables. Doing so begins with identifying characteristic length and velocity scales. The most-commonly-used length scale is the hydraulic diameter, which is defined as

$$
\mathcal{L}_{D_{\mathrm{h}}}=\frac{4 A_{\mathrm{c}}}{P}
$$

where $A_{\mathrm{c}}=a b-\pi d^{2} / 4$ and $P=2 a+2 b+\pi d$ are the channel cross-sectional area and wetted perimeter, respectively. Represented in terms of the aspect ratios,

$$
\mathcal{L}_{D_{\mathrm{h}}}=b \frac{4 \alpha-\pi \beta^{2}}{2(1+\alpha)+\pi \beta} .
$$


Although the hydraulic diameter is widely used, Muzychka and Yovanovich [17] suggest that the square root of the cross-sectional area is a more appropriate characteristic length scale for complex channel geometries. That is,

$$
\mathcal{L}_{\sqrt{A_{\mathrm{c}}}}=\sqrt{A_{\mathrm{c}}}=\sqrt{a b-\frac{\pi d^{2}}{4}}=b \sqrt{\alpha-\frac{\pi \beta^{2}}{4}} .
$$

The velocity scale is taken to be the mean velocity $U$. The mean velocity is related to the net mass-flow rate through the channel as $\dot{m}=\rho U A_{\mathrm{c}}$, where $\rho$ is the fluid's mass density. Using these characteristic scales, dimensionless variables may be written as

$$
\hat{x}=\frac{x}{\mathcal{L}}, \quad \hat{y}=\frac{y}{\mathcal{L}}, \quad \hat{u}=\frac{u}{U} .
$$

At this point, the characteristic length scale is represented as $\mathcal{L}$, without being specific as to using $\mathcal{L}=D_{\mathrm{h}}$ or $\mathcal{L}=\sqrt{A_{\mathrm{c}}}$.

\subsection{Dimensionless equations}

Using the characteristic scales, the dimensionless momentum equation may be written as

$$
\nabla^{2} \hat{u}=\frac{1}{\mu} \frac{\mathcal{L}^{2}}{U} \frac{\mathrm{d} p}{\mathrm{~d} z}
$$

For the sake of simplifying the nomenclature, the variable $\Gamma$ is defined as

$$
\Gamma \equiv \frac{1}{\mu} \frac{\mathcal{L}^{2}}{U} \frac{\mathrm{d} p}{\mathrm{~d} z}
$$

The mean velocity $U$ can be represented in terms of the local velocity distribution as

$$
U=\frac{1}{A_{\mathrm{c}}} \int_{A_{\mathrm{c}}} u \mathrm{~d} A_{\mathrm{c}}
$$


Recognizing that $\hat{A}_{\mathrm{c}}=A_{\mathrm{c}} / \mathcal{L}^{2}$, transformation to dimensionless variables yields

$$
1=\frac{1}{\hat{A}_{\mathrm{c}}} \int_{\hat{A}_{\mathrm{c}}} \hat{u} \mathrm{~d} \hat{A}_{\mathrm{c}} .
$$

Equation 10 represents an integral constraint on the solution of Eq. 7. The parameter $\Gamma$ plays the role of an eigenvalue that must be determined such that Eq. 10 is satisfied. In other words, Eq. 7 has a solution for any value of $\Gamma$. However, the only relevant solution is the one for which $\Gamma$ is chosen such that Eq. 10 is satisfied.

Because $\Gamma$ (right hand side of Eq. (7)) is a constant, it is convenient to define a variable $\phi$ as

$$
\phi \equiv \frac{\hat{u}}{\Gamma} .
$$

Using this definition, the momentum equation can be rewritten as

$$
\nabla^{2} \phi=\nabla^{2}\left(\frac{\hat{u}}{\Gamma}\right)=1 .
$$

Equation 12 is a linear elliptic equation that, in principle, can be solved analytically by separation of variables, even on a complex geometry. However, the analytic solution is difficult to derive, with the result being represented as a very complicated infinite series. Such an analytic solution does not offer higher accuracy or other benefits, and a computational solution is much more easily accomplished. The approach here is to solve the equation computationally on a finite-element mesh using the PDE toolbox in MATLAB (cf., Sect. 2.4). This approach produces highly accurate solutions with very little computational effort.

By using Eqs. 10 and 11, the relevant value for $\Gamma$ can then be extracted 
124

from the solution for $\phi$ as

$$
\Gamma=\frac{\hat{A}_{\mathrm{c}}}{\int_{\hat{A}_{\mathrm{c}}} \phi \mathrm{d} \hat{A}_{\mathrm{c}}} .
$$

125

127

133

134

The full dimensionless area may be evaluated directly as

$$
\hat{A}_{\mathrm{c}}=\frac{A_{\mathrm{c}}}{\mathcal{L}^{2}}=\frac{\left(a b-\pi d^{2} / 4\right)}{\mathcal{L}^{2}}=\frac{b^{2}\left(\alpha-\pi \beta^{2} / 4\right)}{\mathcal{L}^{2}} .
$$

Alternatively, it may be evaluated computationally from the finite-element mesh as

$$
\hat{A}_{\mathrm{c}}=\int_{\hat{A}_{\mathrm{c}}} \mathrm{d} \hat{A}_{\mathrm{c}}
$$

which, by comparing the two values, serves as a check on the accuracy of the numerical quadrature.

The dimensionless momentum equation must be solved on the dimensionless domain (cf., Fig. 2). Using the definition of the characteristic length scale, the dimensionless domain dimensions are

$$
\hat{b}=\frac{b}{\mathcal{L}}
$$

$$
\begin{aligned}
& \hat{a}=\frac{a}{\mathcal{L}}=\alpha \frac{b}{\mathcal{L}}=\alpha \hat{b}, \\
& \hat{d}=\frac{d}{\mathcal{L}}=\beta \frac{b}{\mathcal{L}}=\beta \hat{b} .
\end{aligned}
$$

Note that the dimensionless domain can be written entirely in terms of the dimensionless aspect ratios $\alpha$ and $\beta$ (i.e., Eq. 4 or 5). Thus, different channel aspect ratios lead to different dimensionless domains (cf., Fig. 2) for the solution of Eq. 7.

\subsection{Friction factor}

As is well known and widely practiced, dimensionless measures of mean wall shear stress within channels and ducts can be represented as the product 
of Reynolds number and friction factor, Ref. The general approach to deriving and using Re $f$ correlations can be found in most fluid-mechanics books that deal with internal flows (cf., [14-16]). The objective of the present paper is to derive and evaluate new friction-factor correlations for rectangular channels with interior cylindrical bodies.

The mean shear stress $\tau_{\mathrm{m}}$ associated with fluid-mechanical drag at the channel walls and the probe surface can be written in integral form as

$$
\tau_{\mathrm{m}}=\frac{1}{P} \int_{P} \mu \nabla u \cdot \mathbf{n} \mathrm{d} s
$$

where $s$ is the distance around the perimeter, $P=2 a+2 b+\pi d$ is the wetted perimeter, and $\mathbf{n}$ is an outward-pointing normal unit vector at the solid surfaces around the perimeter. The mean shear stress can be written in dimensionless form using a friction factor $f$ that is defined as

$$
f \equiv \frac{\tau_{\mathrm{m}}}{\frac{1}{2} \rho U^{2}}
$$

It follows directly that

$$
f=\frac{2}{\rho U^{2}}\left(\frac{1}{P} \int_{P} \mu \nabla u \cdot \mathbf{n} \mathrm{d} s\right)
$$

The right-hand side of Eq. 21 can be transformed to dimensionless variables, thus enabling the direct use of the dimensionless solution to the momentum equation (Eq. 7). Considering the definition of the Reynolds number as

$$
\operatorname{Re}=\frac{\rho U \mathcal{L}}{\mu}
$$

an expression for $\operatorname{Re} f$ follows as

$$
f=\frac{2}{\rho U^{2}} \frac{\mu U}{\mathcal{L}}\left(\frac{1}{\hat{P}} \int_{\hat{P}} \nabla \hat{u} \cdot \mathbf{n} \mathrm{d} \hat{s}\right),
$$


158

159

$$
f=\frac{2}{\operatorname{Re}}\left(\frac{1}{\hat{P}} \int_{\hat{P}} \nabla \hat{u} \cdot \mathbf{n d} \hat{s}\right)
$$

$$
\operatorname{Re} f=\frac{2}{\hat{P}} \int_{\hat{P}} \nabla \hat{u} \cdot \mathbf{n} \mathrm{d} \hat{s} .
$$

The dimensionless perimeter may be written as

$$
\hat{P}=\frac{P}{\mathcal{L}}=\frac{b}{\mathcal{L}}(2(1+\alpha)+\pi \beta) .
$$

For any solution of the momentum equation (i.e., $\hat{u}(\hat{x}, \hat{y})$ ), it is evident from Eq. 25 that $\operatorname{Re} f$ is a constant. Although evaluating the perimeter integral (Eq. 25) can be accomplished computationally, it can be cumbersome for the complex geometry. Thus, as explained in the following paragraph, an easier-to-implement alternative can be developed.

A force balance on an axial differential section of the fluid within the rectangular channel can be written as

$$
A_{\mathrm{c}} \frac{\mathrm{d} p}{\mathrm{~d} z}=P \tau_{\mathrm{m}}=P \frac{1}{P} \int_{P} \mu \nabla u \cdot \mathbf{n} \mathrm{d} s .
$$

In other words, the pressure gradient acting on the fluid cross section is balanced by the shear drag associated with the channel walls and the cylindrical probe surface. Transforming to dimensionless variables yields

$$
\frac{\mathcal{L}^{2}}{\mu U} \frac{\mathrm{d} p}{\mathrm{~d} z}=\frac{1}{\hat{A}_{\mathrm{c}}} \int_{\hat{P}} \nabla \hat{u} \cdot \mathbf{n} \mathrm{d} \hat{s} .
$$

Equation 25 can be substituted for the perimeter integral, yielding

$$
\frac{\mathcal{L}^{2}}{\mu U} \frac{\mathrm{d} p}{\mathrm{~d} z}=\frac{1}{2} \frac{\hat{P}}{\hat{A}_{\mathrm{c}}} \operatorname{Re} f .
$$

172 Using the definition of the eigenvalue $\Gamma$ (Eq. 8),

$$
\operatorname{Re} f=\frac{\mathcal{L}^{2}}{\mu U} \frac{\mathrm{d} p}{\mathrm{~d} z} \frac{2 \hat{A}_{\mathrm{c}}}{\hat{P}}=\frac{2 \hat{A}_{\mathrm{c}}}{\hat{P}^{2}} \Gamma .
$$


The dimensionless cross-sectional area and perimeter are stated in Eqs. 14 and 26, respectively. Thus, once a geometry is specified and the dimensionless momentum equation is solved, establishing the eigenvalue $\Gamma$, the value of the constant Ref follows directly. Using the hydraulic diameter as the characteristic length scale (Eq. 4) and using Eqs. 14 and 26 for $\hat{A}_{\mathrm{c}}$ and $\hat{P}$, respectively, it follows that $\hat{A}_{\mathrm{c}} / \hat{P}=1 / 4$. Thus, Eq. 30 simplifies as

$$
\operatorname{Re} f_{D_{\mathrm{h}}}=\frac{\Gamma}{2} .
$$

The Reynolds number in the analysis, and hence in Eq. 30, is based on the characteristic length scale $\mathcal{L}$. However, as discussed earlier, the characteristic length may be defined alternatively as the hydraulic diameter $\mathcal{L}_{D_{\mathrm{h}}}$ or the square root of the cross-sectional area $\mathcal{L}_{\sqrt{A_{\mathrm{c}}}}$. Depending on the length-scale definition, the dimensionless computational domain changes. Furthermore, the interpretation of $\operatorname{Re} f$ depends on the length-scale definition (i.e., $\operatorname{Re} f_{D_{\mathrm{h}}}$ or $\left.\operatorname{Re} f_{\sqrt{A_{\mathrm{c}}}}\right)$.

[Figure 3 about here.]

\subsection{Computational Procedure}

Equation 12 is a linear equation that is solved computationally within the Matlab PDE toolbox using a finite-element method with adaptive mesh refinement. High accuracy is assured by mesh refinement to the point that the solutions are independent of mesh resolution. The solutions use between 10,000 and 100,000 finite elements. Each solution requires only a few seconds on a typical personal computer.

The equations are solved numerous times for different probe placements and aspect ratios. Figure 3 shows three representative solutions, each with 
a different probe placement. In all these cases, the aspect ratios are $\alpha=1$ and $\beta=0.2$. The contours show scaled dimensionless velocity maps. It is evident that the velocities are much lower (hence more shear drag) when the probe is placed in the center of the channel. Consequently, the mean velocity (Eq. 9) is lower when the cylinder is more centrally located.

[Figure 4 about here.]

[Figure 5 about here.]

\section{3. $\operatorname{Re} f$ correlations}

Figure 4 shows $\operatorname{Re} f_{\mathrm{D}_{\mathrm{h}}}$ correlations as functions of the cylinder aspect ratio $\beta(\beta>0.001)$ for a range of rectangular-channel aspect ratios $\alpha$. In all cases, the cylinder is at the center of the rectangular channel. At very small values of $\beta$ the $\operatorname{Re} f_{\mathrm{D}_{\mathrm{h}}}$ correlation becomes very steep.

When $\beta=0$, the central cylinder disappears. Thus, the no-slip (zerovelocity) boundary condition at the cylinder surface no longer applies. Consequently, the problem becomes singular if a no-slip boundary condition is applied at a surface that does not exist. As discussed subsequently, the problem can be solved without an interior cylindrical body (e.g., probe), yielding, for example, the well-known result of $\operatorname{Re} f_{\mathrm{D}_{\mathrm{h}}}=14.22$ when $\alpha=1$.

The correlations shown in Fig. 4 have some possibly unanticipated characteristics, which result from the definitions of the length scales in the dimensionless analysis. The $\operatorname{Re} f_{\mathrm{D}_{\mathrm{h}}}$ functions all reach a maximum value at some $\beta$, and then decrease at high $\beta$. As $\beta$ increases, the cylinder occupies a larger fraction of the channel. Thus, as the flow area decreases the net shear stress 
and pressure drop must increase for particular mass flow rates. However, it is interesting that $\operatorname{Re} f_{\mathrm{D}_{\mathrm{h}}}$ decreases as $\beta$ increases toward its maximum value (i.e., where cylinder touches a channel wall in dimensionless space). At $\beta \approx 0.055$, the functions all cross, with $\operatorname{Re} f_{\mathrm{D}_{\mathrm{h}}}$ being essentially independent of $\alpha$. The functional forms of $\operatorname{Re} f_{\mathrm{D}_{\mathrm{h}}}$ are the result of the hydraulic-diameter definition and the dimensionless analysis. Despite the fact that the dimensionless $\operatorname{Re} f_{\mathrm{D}_{\mathrm{h}}}$ functions have local maxima (Figs. 4-7), the physical behaviors do not have such local maxima. The examples discussed in Sect. 4 show the expected behaviors. That is, for example, as the cylinder becomes larger the pressure drop increases monotonically.

Figure 5 shows $\operatorname{Re} f_{\mathrm{D}_{\mathrm{h}}}$ correlations as functions of the cylinder aspect ratio $\beta$ for a range of rectangular-channel aspect ratios $\alpha$. In all cases, the cylinder is in the corner of the rectangular channel. Compared to Fig. 4, the $\operatorname{Re} f_{\mathrm{D}_{\mathrm{h}}}$ functions are qualitatively very different. Placing the cylinder in the corner leads to $\operatorname{Re} f_{\mathrm{D}_{\mathrm{h}}}$ mostly decreasing monotonically as a function of $\beta$. However, there are weak local minima for $\alpha>0.8$ and large $\beta$. For most of the $\beta$ range, the $\operatorname{Re} f_{\mathrm{D}_{\mathrm{h}}}$ values are lower for the corner cases than they are for the centrally placed cylinder.

Figure 6 shows $\operatorname{Re} f_{\mathrm{D}_{\mathrm{h}}}$ correlations as functions of the cylinder aspect ratio $\beta$ for a range of rectangular-channel aspect ratios $\alpha$. In all cases, the cylinder is on the middle of the floor of the rectangular channel. Again, the correlations are qualitatively different from those shown in Figs. 4 and 5.

Although Figs. 4-6 show correlations for wide ranges of the rectangularchannel aspect ratios, practical catalyst monoliths almost always use square channels (i.e., $\alpha=1$ ). Figure 7 shows correlations for $\alpha=1$, but with 
different cylinder diameters $\beta$ and placements.

At $\beta=0$ (i.e., open channel without a cylinder), the results show $\operatorname{Re} f_{\mathrm{D}_{\mathrm{h}}} \approx$ 14.22 , which is the well-known result for fully developed flow in a square channel $[14,15]$. At large $\beta$, where the cylinder nearly touches the channel walls, the results for all three cylinder positions approach $\operatorname{Re} f_{\mathrm{D}_{\mathrm{h}}} \approx 8$. From the viewpoint of monolith-sampling technology, this result (i.e., the cylinder filling the channel as much as possible) has relatively little practical utility. However, there may well be other circumstances in which a cylinder that fills a rectangular channel is relevant. Table 1 lists polynomial fitting coefficients that replicate the results $(\alpha=1)$ that are shown graphically in Fig. 7 .

As $\beta$ approaches zero (i.e., the cylindrical probe vanishes), the asymptotic behavior of $\operatorname{Re} f$ is qualitatively different in the case of the centrally placed cylinder than it is when the cylinder is adjacent to a channel wall. A no-slip boundary condition is applied to the cylinder wall, which serves to retard the flow in the vicinity of the cylinder. For centrally positioned cylinders, even with very small cylinder diameters (i.e., small $\beta$ ), the no-slip condition retards the flow in the central regions of the channel. At $\beta=0$ (i.e., without a cylinder) the no-slip boundary condition at the cylinder wall is no longer applicable and the problem must be solved for the channel alone without a cylinder. In other words, the fundamental problem formulation and boundary conditions must change at $\beta=0$. However, when the cylinder is adjacent to the channel wall (bottom and corner cases), the situation is different. As the cylinder diameter becomes smaller and smaller, the no-slip boundary condition still applies. However, in this case the no-slip boundary condition at the cylinder surface becomes indistinguishable from the no-slip boundary 
condition at the cylinder wall. Thus, the $\operatorname{Re} f$ solutions smoothly approach the open-channel solutions.

Although the shapes of the Re $f$ correlations shown in Fig. 7 are different, the reasons for the common end points are evident. When the cylinder vanishes (i.e., $\beta=0$ ), all the solutions must converge to the open-channel solution (i.e., $\operatorname{Re} f=14.22$ ). When the cylinder becomes as large as possible (i.e., touching the channel walls), again all the $\operatorname{Re} f$ solutions must be the same. Regardless of whether the cylinder begins in the center, at the corner, or on the mid bottom, for $\alpha=1$ the geometries are identical when $\beta=1$. The only open flow spaces are the approximately triangular regions in the channel corners. The correlation $\operatorname{Re} f_{\mathrm{D}_{\mathrm{h}}} \approx 8$ of this particular geometric configuration is a new result of the present paper.

[Figure 6 about here.]

[Figure 7 about here.]

[Figure 8 about here.]

\subsection{Prior $\operatorname{Ref}$ correlations}

Figure 8 illustrates $\operatorname{Re} f$ correlations for the well-known cases of flow in open rectangular channels and cylindrical-annulus ducts $[14,15]$. In both cases the Reynolds number is based on hydraulic diameter. In open rectangular channels the correlation decreases monotonically from $\operatorname{Re} f=24$ to approximately $\operatorname{Re} f \approx 14.2$. In the annular case, the correlation function increases monotonically from $\operatorname{Re} f=16$ (circular duct without a center cylinder) to $\operatorname{Re} f=24$ (asymptotic result for parallel plates). The inset equations 
show analytic expressions for the correlations. The relatively simple expression for the open rectangular channel was taken from Kee, et al. [18]; a moreaccurate five-parameter polynomial fit can be found in Shah and Sekulić [14].

Intuitively, one might expect the functional behaviors seen in Figs. 47 to be similar to those seen in Fig. 8. However, with two independent geometric parameters $(\alpha$ and $\beta)$, the correlations are inherently more complex than those of the well-known simpler geometric configurations with only one geometric parameter (e.g., Fig. 8)

\subsection{Length scale based on $\sqrt{A_{\mathrm{c}}}$}

Dimensionless analysis necessarily depends on characteristic scales. The hydraulic diameter (Eq. 3) is by far the most commonly used length scale for analyzing flow in channels and ducts. However, there is no requirement that any particular scale be used. Alternative characteristic scales may lead to better or more-easily-used correlations. Muzychka and Yovanovich [17] suggest advantages associated with using the square root of the cross-sectional area as the characteristic length scale. Thus, it is reasonable to investigate the possibility that $\mathcal{L}=\sqrt{A_{\mathrm{c}}}$ might produce simpler or more intuitive function forms for the $\operatorname{Re} f$ correlations.

Using $\mathcal{L}=\sqrt{A_{\mathrm{c}}}$, Fig. 9 shows $\operatorname{Re} f_{\sqrt{A_{\mathrm{c}}}}$ as functions of $\beta$ for the square channel cross sections. The functional forms of $\operatorname{Re} f_{\sqrt{A_{\mathrm{c}}}}$ are significantly different from function forms for $\operatorname{Re} f_{D_{\mathrm{h}}}$ based on the hydraulic diameter as shown in Fig. 7. However, both correlations yield relatively complicated nonlinear functional forms for Ref. Thus, with no apparent advantage for using $\mathcal{L}=\sqrt{A_{\mathrm{c}}}$, the paper presents the results in terms of the hydraulic diameter being the characteristic length scale. Of course, the physical results 
(e.g., pressure drops) are independent of the characteristic length scales that are used in formulating the dimensionless problem.

[Table 1 about here.]

[Figure 9 about here.]

\subsection{Entry region}

The foregoing analysis is based upon fully developed flow. However, in fact, as uniform flow enters a channel, boundary layers form on the channel walls. The flow becomes fully developed only after some entry-length region. In the entry region, the friction factor is higher than it is once the flow becomes fully developed. Thus, it is important to evaluate the validity associated with assuming fully developed flow.

Muzychka and Yovanovich [17] develop a general analysis to predict the variation of $\operatorname{Re} f$ in the entry regions of a wide range of noncircular ducts. The general expression is written as

$$
\operatorname{Re} f_{\sqrt{A_{\mathrm{c}}}}=\sqrt{\left(\frac{3.44}{\sqrt{L^{+}}}\right)^{2}+\left(\frac{12}{\sqrt{\varepsilon}(1+\varepsilon)\left[1-\frac{192 \varepsilon}{\pi^{5}} \tanh \left(\frac{\pi}{2 \varepsilon}\right)\right]}\right)^{2}} .
$$

where the dimensionless length from the channel entrance is

$$
L^{+}=\frac{L}{\sqrt{A_{\mathrm{c}}}} \frac{1}{\operatorname{Re}_{\sqrt{A_{\mathrm{c}}}}},
$$

and $\varepsilon$ is a general representation of the channel aspect ratio. However, $\varepsilon$ as defined by Muzychka and Yovanovich is different from the aspect ratios $\alpha$ and $\beta$. For channels such as the ones considered here, there is no precise definition of $\varepsilon$. Nevertheless, Muzychka and Yovanovich suggest an approximation as

$$
\varepsilon=\frac{1-\sqrt{A_{\mathrm{i}} / A_{\mathrm{o}}}}{\pi\left(1+\sqrt{A_{\mathrm{i}} / A_{\mathrm{o}}}\right)}
$$


where $A_{\mathrm{i}}$ and $A_{\mathrm{o}}$ are the areas of inner and outer "polygons" that bound the flow area. Here, $A_{\mathrm{i}}=\pi d^{2} / 4$ and $A_{\mathrm{o}}=a \times b$. Substituting the definitions for $\alpha$ and $\beta$, the area ratio in Eq. 34 can be rewritten as

$$
\sqrt{\frac{A_{\mathrm{i}}}{A_{\mathrm{o}}}}=\sqrt{\frac{\pi}{4} \frac{\beta^{2}}{\alpha}} .
$$

Based on Eq. 32, Fig. 10 shows predicted Re $f$ as a function of $L^{+}$for two different aspect ratios $\varepsilon$. The asymptotic fully developed value of $\operatorname{Re} f$ is reached at $L^{+} \approx 0.1$. Thus, the actual flow-development length depends on the Reynolds number, with lower Reynolds numbers leading to more-rapid approach to the fully developed flow.

Because of relatively small characteristic diameters and low flow rates, small channel devices tend to operate at low Reynolds numbers. For example, at $R e=10$, the entry-length region is only about one channel diameter. At $\operatorname{Re}=100$, the entry-length region is approximately ten channel diameters. Thus, although some caution needs to be exercised, the fully developed solutions are expected to have practical utility, even in relatively short channels and ducts.

[Figure 10 about here.]

Consider for example, the partial-oxidation catalyst monolith reported by Diehm and Deutschmann [10]. The monolith had 600 square cells per square inch, leading to individual channel dimensions of approximately $1 \mathrm{~mm}$. The inlet flow velocities were approximately $1 \mathrm{~m} \mathrm{~s}^{-1}$. Assuming properties of air at $900 \mathrm{~K}$, the viscosity is $\mu \approx 4 \times 10^{-5} \mathrm{~Pa}$ s and the mass density is $\rho=0.4 \mathrm{~kg} \mathrm{~m}^{-3}$. These conditions lead to a Reynolds number of $\mathrm{Re} \approx 10$. 
Thus, even for relatively high flow rates associated with partial-oxidation catalysts, the hydrodynamic entry length is only about one channel diameter. The monolith reported by Diehm and Deutschmann was $11 \mathrm{~mm}$ long. Thus, approximately $90 \%$ of the channel was in the fully developed flow regime. The analysis presented in this paper is generally consistent with the relatively more complicated three-dimensional computational fluid dynamics simulations by Hettel et al. [9].

\section{Illustrative examples}

Illustrative examples serve to demonstrate the use of the correlations. Continue to consider the partial-oxidation catalyst monolith reported by Diehm and Deutschmann [10]. The monolith had 600 square cells per square inch, resulting in individual channel dimensions of approximately $a=b=1$ $\mathrm{mm}(\alpha=1)$. For an open square channel, $\operatorname{Re} f_{D_{\mathrm{h}}}=14.2[15]$. Using the reported mean flow velocities of approximately $U=1 \mathrm{~m} \mathrm{~s}^{-1}$ and assuming properties of air at $900 \mathrm{~K}\left(\mu \approx 4 \times 10^{-5} \mathrm{~Pa} \mathrm{~s}\right.$ and $\left.\rho=0.4 \mathrm{~kg} \mathrm{~m}^{-3}\right)$, the Reynolds number for a single monolith channel is

$$
\operatorname{Re}=\frac{\rho U D_{\mathrm{h}}}{\mu}=10,
$$

where the hydraulic diameter is $D_{\mathrm{h}}=4 A_{\mathrm{c}} / P=1 \mathrm{~mm}$. The friction factor easily follows as

$$
f=\frac{\operatorname{Re} f}{\operatorname{Re}}=1.42 .
$$

The mean shear stress can be evaluated (Eq. 21) as

$$
\tau_{\mathrm{m}}=\frac{1}{2} \rho U^{2} f=0.284 \mathrm{~Pa}
$$


The force balance on an axial section of the fluid within the channel can be written as

$$
A_{\mathrm{c}} \frac{\mathrm{d} p}{\mathrm{~d} z}=P \tau_{\mathrm{m}},
$$

where $A_{\mathrm{c}}=1 \mathrm{~mm}^{2}$ and $P=4 \mathrm{~mm}$ are the channel cross-sectional area and wetted perimeter, respectively. For a channel length of $L=20 \mathrm{~mm}$, the predicted pressure drop is $23 \mathrm{~Pa}$. It is reasonable to assume that each of the unobstructed channels in the monolith experience the same pressure drop. Thus the pressure difference across the monolith is $\Delta p=23 \mathrm{~Pa}$.

Assume now that a cylindrical probe is placed within a single channel of the monolith. The pressure drop is fixed, but shear stress will increase and the velocity will decrease. Diehm and Deutschmann [10] used a $170 \mu \mathrm{m}$ diameter capillary probe $(\beta=0.17)$. Using either Fig. 4 or Table 1 , with $\alpha=1, \beta=0.17$ and a centered probe, $\operatorname{Re} f \approx 20.7$. Using a rearrangement of Eq. 30, the mean velocity for the obstructed channel is reduced from $U=1$ $\mathrm{m} \mathrm{s}^{-1}$ to

$$
U=2 \frac{\Delta p}{L} \frac{A_{\mathrm{c}}}{P} \frac{D_{\mathrm{h}}}{\mu \operatorname{Re} f}=0.5 \mathrm{~m} \mathrm{~s}^{-1},
$$

where the definitions of $P, A_{\mathrm{c}}$, and $D_{\mathrm{h}}$ now include the probe. The velocity in the obstructed channel is half of that of the other channels.

Consider now the case where the probe lies in the corner of the channel, not in the center. Using Fig. 4 or Table $1, \operatorname{Re} f=10.8$. In this case, the velocity decrement is very small at $U=0.98 \mathrm{~m} \mathrm{~s}^{-1}$. Thus, it is clear that in this application a corner-placed probe produces only a minimal perturbation of the flow, and hence pressure drop. Placing the probe on the bottom middle of the channel floor also produces only a small flow perturbation. Of course, as a practical matter, probe placement in any particular experiment may 
need to consider other factors beyond flow perturbation and shear drag.

Diehm and Deutschmann [10] also used a $630 \mu \mathrm{m}$ diameter sheathed thermocouple, leading to $\beta=0.63$. In this case, the center-position probe leads to $\operatorname{Re} f=21.8$ and a mean velocity of $U=0.138 \mathrm{~m} \mathrm{~s}^{-1}$. The cornerposition probe leads to $\operatorname{Re} f=8.35$ and a higher mean velocity of $U=0.36$ $\mathrm{m} \mathrm{s}^{-1}$.

Figure 11 summarizes the effect of probe diameter and position on the mean velocity for this example. Clearly, the corner position is the least intrusive, followed by the middle-bottom position, and then the center position. Alternatively, for a specified mass flow rate through the channel, the corner probe requires a lower pressure drop than is required for the center of middle probe positions. As the probe diameter increases, the velocity for all positions decreases. For a square channel $(\alpha=1)$, as the probe entirely fills the channel (i.e., $\beta=1$ ), all the probe "positions" approach being the same.

Finally, consider the center-cylinder correlations shown in Fig. 4, where all the correlating functions cross at $\beta \approx 0.055$. Figure 12 shows two channels with different aspect ratios, but with the same centered cylinder diameter. Both have a horizontal dimension of $b=2 \mathrm{~mm}$ and a cylinder diameter of $d=0.11 \mathrm{~mm}$. The upper channel has aspect ratio $\alpha=1$ and the lower channel has aspect ratio $\alpha=0.1$. Thus, referring to Fig. 4 , it is evident that these two channels are both at the crossing point where $\operatorname{Re} f \approx 19$. Although the friction factor correlations are identical, it is clear that the flow behaviors for these channels must be quite different. Assume further that both channels are $50 \mathrm{~mm}$ long and that a $50 \mathrm{~Pa}$ pressure difference is imposed between the channel entrances and exits. Continuing to use the properties of air at 900 
K, Eq. 40 predicts mean velocities of $U=2.4 \mathrm{~m} \mathrm{~s}^{-1}$ for the larger $\alpha=1$ channel and $U=0.07 \mathrm{~m} \mathrm{~s}^{-1}$ for the smaller $\alpha=0.1$ channel.

[Figure 11 about here.]

[Figure 12 about here.]

\section{Plug-flow models}

An important application of friction-factor correlations is to evaluate local wall shear stress in low-dimension plug-flow models. The friction-factor correlations themselves are derived with two-dimensional models, assuming indefinitely long channels and correspondingly long central cylinders. In practice, finite-length probes may be inserted into channels. In other words, some portion of the channel may contain a probe cylinder and other portions may not. Moreover, in models that also include thermal and chemical behavior, the mass density $\rho$ and viscosity $\mu$ may vary axially along the channel length.

Along the length of a channel, a steady-state plug-flow momentum equation can be represented as

$$
\frac{\mathrm{d}\left(\rho U^{2}\right)}{\mathrm{d} z}=-\frac{\mathrm{d} p}{\mathrm{~d} z}-\tau_{\mathrm{m}} \frac{P}{A_{\mathrm{c}}}
$$

where $\rho$ is the mass density, $U$ is the mean velocity, $p$ is the pressure, $\tau_{\mathrm{m}}$ is the local mean wall shear stress, $P$ is the wetted perimeter, and $A_{\mathrm{c}}$ is the crosssectional flow area. The Re $f$ correlations may be used to evaluate the local wall shear stress. The local Reynolds number (Eq. 22) can be evaluated in terms of the local mean velocity $U$ (i.e., the dependent variable of the 
momentum equation). Thus, knowing the local friction-factor correlation, the local friction-factor itself is evaluated as

$$
f=\frac{\operatorname{Re} f}{\operatorname{Re}}
$$

where $\operatorname{Re} f$ is a constant evaluated from the correlations. As the geometry varies along the length of the channel (e.g., with or without a probe), the Ref correlation varies. Moreover, if the viscosity or density vary axially, then the local Reynolds number varies axially. The local mean shear stress is evaluated from the local friction factor using Eq. 21. The plug-flow model does not account for "entry length" transitions between abrupt changes in the local channel configuration. However, for low Reynolds number flows the transitions are rapid (cf., Fig. 10).

\section{Summary and conclusions}

Motivated by a need to understand how in-situ diagnostic probes affect flow through small channels, the present analysis develops general frictionfactor correlations for steady-state, fully developed, laminar flow in rectangular channels with interior cylindrical bodies. The analysis incorporates the influence of alternative flow conditions and geometrical configurations (i.e., channel aspect ratio and ratio of probe diameter and channel width). The correlations are represented in a traditional way as the product of Reynolds number and friction factor Ref, with the characteristic length scale being the hydraulic diameter. The study considers three placements of the interior cylinder. A centrally placed cylinder causes the greatest flow restriction, and is thus most invasive as a diagnostics probe. Locating the cylinder in the 
corner of the rectangular channel causes the least flow disturbance. Placing the probe at the center of the channel floor causes an intermediate flow restriction, but is still much less perturbative than a centrally place probe. The correlations enable direct quantification of a probe that extends through the full length of a channel. In practice, however, probes may be only partially inserted. Using a plug-flow model, the correlations can also be used to evaluate the influence of partially inserted probes and flows where the fluid properties vary along the channel length.

\section{Acknowledgements}

This research was supported by the Office of Naval Research (grant N0001412-1-0201). The effort was initiated during a visit by RJK at the Karlsruhe Institute of Technology (KIT), and completed during a visit by HG at the Colorado School of Mines. Travel expenses for RJK were supported by the Deutsche Forschungsgemeinschaft (DFG) within the framework of the DFG research unit FOR 1993 "Multi-functional conversion of chemical species and energy." HG gratefully acknowledges financial support by the Karlsruhe House of Young Scientists (KHYS) at KIT.

\section{References}

\section{References}

[1] W.P. Partridge, J.M.E. Storey, S.A. Lewis, R.W. Smithwick, G.L. Devault, M.J. Cunningham, N.W. Currier, and T.M. Yonushonis. Timeresolved measurements of emission transients by mass spectrometry. 
SAE Technical Paper 2000-01-2952, SAE International, Warrendale, PA, 2000.

[2] R. Horn, K.A. Williams, N.J. Degenstein, A. Bitsch-Larsen, D. Dalle Nogare, S.A. Tupy, and L.D. Schmidt. Methane catalytic partial oxidation on autothermal Rh and Pt foam catalysts: Oxidation and reforming zones, transport effects, and approach to thermodynamic equilibrium. J. Catalysis, 249(2):380-393, 2007.

[3] O. Shakir, A. Yezerets, N.W. Currier, and W.S. Epling. Spatially resolving concentration and temperature gradients during the oxidation of propylene on Pt/ $\mathrm{Al}_{2} \mathrm{O}_{3}$. Appl. Catal. A: General, 365(2):301-308, 2009.

[4] J. Sá, D.L.A. Fernandes, F. Aiouache, A. Goguet, C. Hardacre, D. Lundie, W. Naeem, W.P. Partridge, and C. Stere. SpaciMS: spatial and temporal operando resolution of reactions within catalytic monoliths. Analyst, 135:2260-2272, 2010.

[5] J.-Y. Luo, X. Hou, P. Wijayakoon, S.J. Schmieg, W. Li, and W.S. Epling. Spatially resolving SCR reactions over a Fe/zeolite catalyst. Appl. Catal. B: Environmental, 102:110-119, 2011.

[6] A. Donazzi, D. Livio, M. Maestri, A. Beretta, G. Groppi, E. Tronconi, and P. Forzatti. Synergy of homogeneous and heterogeneous chemistry probed by in situ spatially resolved measurements of temperature and composition. Angewandte Chemie International Edition, 50(17):3943$3946,2011$. 
[7] V. Easterling, Y. Ji, M. Crocker, M. Dearth, and R.W. McCabe. Application of spaciMS to the study of ammonia formation in lean NOx trap catalysts. Appl. Catal. B: Environmental, 123-124:339-350, 2012.

[8] D. Livio, C. Diehm, A. Donazzi, A. Beretta, and O. Deutschmann. Catalytic partial oxidation of ethanol over $\mathrm{Rh} / \mathrm{Al}_{2} \mathrm{O}_{3}$ : Spatially resolved temperature and concentration profiles. Appl. Catalysis A: General, 467:530-541, 2013.

[9] M. Hettel, C. Diehm, B. Torkashvand, and O. Deutschmann. Critical evaluation of in situ probe techniques for catalytic honeycomb monoliths. Catalysis Today, 216:2-10, 2013.

[10] C. Diehm and O. Deutschmann. Hydrogen production by catalytic partial oxidation of methane over staged $\mathrm{Pd} / \mathrm{Rh}$ coated monoliths: Spatially resolved concentration and temperature profiles. Intl. J. Hydrogen Energy, 39:17998-18004, 2014.

[11] A. Goguet, W.P. Partridge, F. Aiouche, C. Hardacre, K. Morgan, C. Stere, and J. Sá. Comment on "The Critical evaluation of in situ probe techniques for catalytic honeycomb monoliths" by Hettel et al. Catalysis Today, 236, Part B:206-208, 2014.

[12] M. Hettel, C. Diehm, and O. Deutschmann. Answer to the comment from Goguet et al. to the paper "The critical evaluation of in situ probe techniques for catalytic honeycomb monoliths" by Hettel et al. Catalysis Today, pages 209-213, 2014. 
[13] K. Morgan, J. Touitou, J.-S. Choi, C. Coney, C. Hardacre, J.A. Pihl, C.E. Stere, M.-Y. Kim, C. Stewart, A. Goguet, and W.P. Partridge. Evolution and enabling capabilities of spatially resolved techniques for the characterization of heterogeneously catalyzed reactions. ACS Catal., in press, 2016. DOI: 10.1021/acscatal.5b02602.

[14] R.K. Shah and D.P. Sekulić. Fundamentals of Heat Exchanger Design. Wiley, Hoboken, NJ, 2003.

[15] W.M. Kays and M.E. Crawford. Convective heat and mass transfer. McGraw-Hill, Inc., New York, NY, 1993.

[16] R.J. Kee, M.E. Coltrin, and P. Glarborg. Chemically Reacting Flow: Theory and practice. Wiley, Hoboken, NJ, 2003.

[17] Y.S. Muzychka and M.M. Yovanovich. Pressure drop in laminar developing flow in noncircular ducts: A scaling and modeling approach. $J$. Fluids Engineering, 131:11105, 2009.

[18] R.J. Kee, P. Korada, K. Walters, and M. Pavol. A generalized model of flow distribution in channel networks of planar fuel cells. J. Power Sources, 109:148-159, 2002. 


\section{List of Figures}

1 Catalytic monolith with a capillary probe within a single channel. . . . . . . . . . . . . . . . . . . . . 30

2 Channel and probe geometry and layout. The rectangular channel is $a$ high and $b$ wide. The probe has a diameter $d$. All physical dimensions are written in dimensionless form, normalized by the characteristic length $\mathcal{L}$. For example, $\hat{a}=a / \mathcal{L} . \quad 31$

3 Dimensionless velocity contour maps for three cylinder placements. In all cases, the rectangle aspect ratio is $\alpha=1$ and the cylinder aspect ratio is $\beta=0.2$. The dimensionless-velocity scale bar is the same for each solution. . . . . . . . . . . . 32

4 Reynolds-number-friction-factor product $\operatorname{Re} f_{\mathrm{D}_{\mathrm{h}}}$ as a function of the cylinder-diameter aspect ratio $\beta$ for ten values of the rectangular channel aspect ratio $\alpha$. The cylinder is placed in the middle of the rectangular channel. The shaded circles indicate how the cylinder diameter changes as $\beta$ changes. . . . 33

5 Reynolds-number-friction-factor product $\operatorname{Re} f_{\mathrm{D}_{\mathrm{h}}}$ as a function of the cylinder-diameter aspect ratio $\beta$ for ten values of the rectangular channel aspect ratio $\alpha$. The cylinder is placed in the corner of the rectangular channel. The shaded circles indicate how the cylinder diameter changes as $\beta$ changes. . . . 34

6 Reynolds-number-friction-factor product $\operatorname{Re} f_{\mathrm{D}_{\mathrm{h}}}$ as a function of the cylinder-diameter aspect ratio $\beta$ for ten values of the rectangular channel aspect ratio $\alpha$. The cylinder is placed on the middle of the floor of the rectangular channel. The shaded circles indicate how the cylinder diameter changes as $\beta$ changes. 35

7 Reynolds-number-friction-factor product $\operatorname{Re} f_{\mathrm{D}_{\mathrm{h}}}$ as a function of the cylinder-diameter aspect ratio $\beta$ for rectangular-channel aspect ratio $\alpha=1.0$. The cylinder is placed either in the center of the channel, in the corner of the channel, or at the middle of the floor of the rectangular channel. . . . . . . . . . . 36

8 Well-known Ref correlations for fully developed flow in a) open rectangular channels and b) cylindrical annuli. In both cases, the Reynolds number is based on hydraulic diameter. . . 37 
9 Reynolds-number-friction-factor product $\operatorname{Re} f_{\sqrt{A_{c}}}$ as a function of the cylinder-diameter aspect ratio $\beta$ for rectangular-channel aspect ratio $\alpha=1.0$. The cylinder is placed either in the center of the channel, in the corner of the channel, or at the middle of the floor of the rectangular channel. . . . . . . . . . . . 38

$10 \operatorname{Re} f$ as a function of $L^{+}$as predicted from Eq. 32 for two different aspect ratios. . . . . . . . . . . . . . . . . . . 39

11 Mean inlet velocity as a function of cylinder aspect ratio $\beta$ for three probe positions. The pressure drop across a $20 \mathrm{~mm}$ channel length is fixed at $\Delta p=23 \mathrm{~Pa}$. The square channel is $1 \mathrm{~mm}$ on a side $(\alpha=1)$, and fluid properties of air at $900{ }^{\circ} \mathrm{C}$ are assumed. . . . . . . . . . . . . . . . . . . . 40

12 Two rectangular channels with a centered cylinder, both having $\beta \approx 0.055$. The upper channel has aspect ratio $\alpha=1$ and the lower channel has aspect ratio $\alpha=0.1 . \ldots 4$. . . . . . 41 


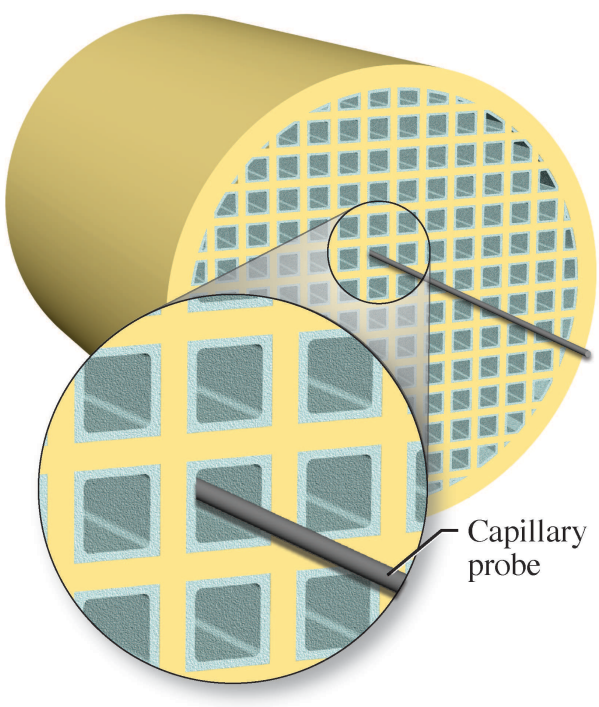

Figure 1: Catalytic monolith with a capillary probe within a single channel. 


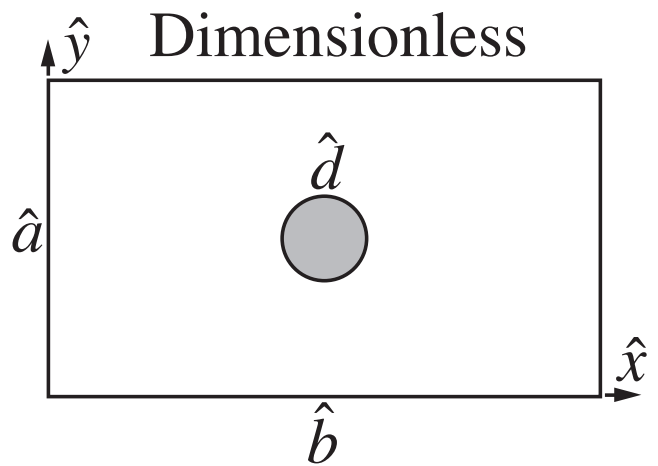

Figure 2: Channel and probe geometry and layout. The rectangular channel is $a$ high and $b$ wide. The probe has a diameter $d$. All physical dimensions are written in dimensionless form, normalized by the characteristic length $\mathcal{L}$. For example, $\hat{a}=a / \mathcal{L}$. 

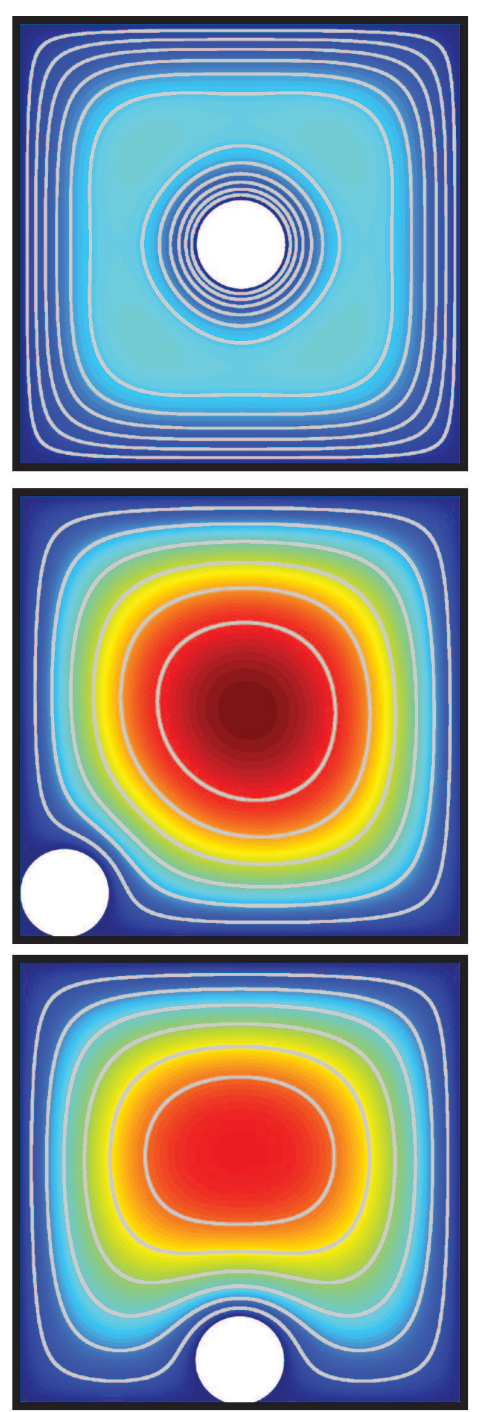

Scaled velocity

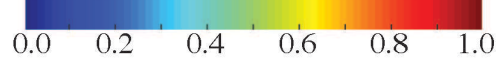

Figure 3: Dimensionless velocity contour maps for three cylinder placements. In all cases, the rectangle aspect ratio is $\alpha=1$ and the cylinder aspect ratio is $\beta=0.2$. The dimensionless-velocity scale bar is the same for each solution. 

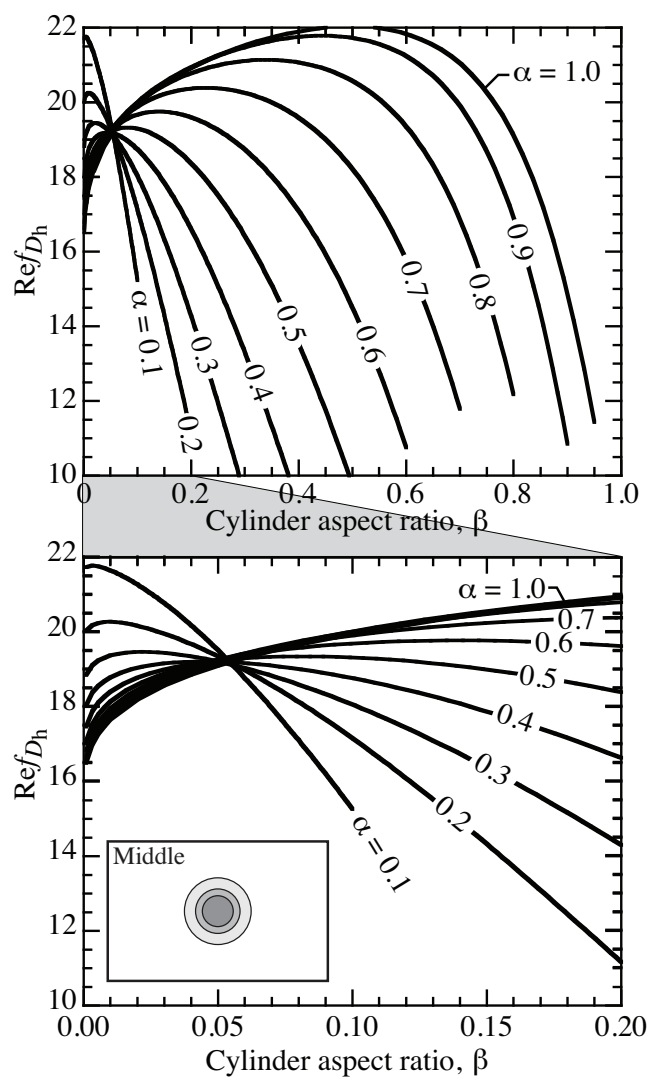

Figure 4: Reynolds-number-friction-factor product $\operatorname{Re} f_{\mathrm{D}_{h}}$ as a function of the cylinderdiameter aspect ratio $\beta$ for ten values of the rectangular channel aspect ratio $\alpha$. The cylinder is placed in the middle of the rectangular channel. The shaded circles indicate how the cylinder diameter changes as $\beta$ changes. 

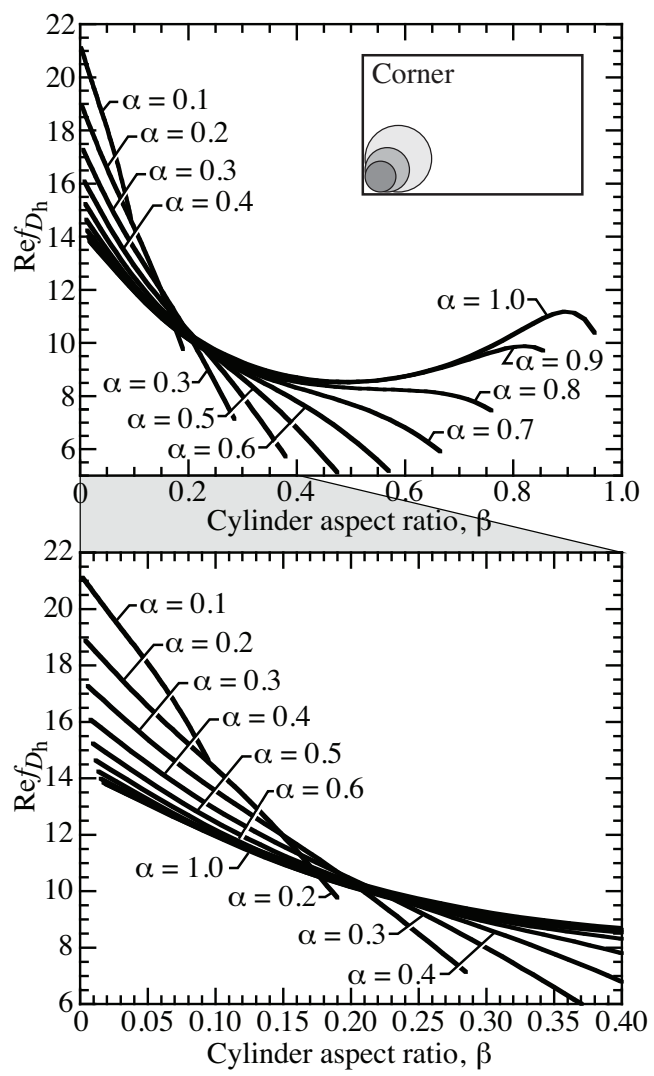

Figure 5: Reynolds-number-friction-factor product $\operatorname{Re} f_{\mathrm{D}_{\mathrm{h}}}$ as a function of the cylinderdiameter aspect ratio $\beta$ for ten values of the rectangular channel aspect ratio $\alpha$. The cylinder is placed in the corner of the rectangular channel. The shaded circles indicate how the cylinder diameter changes as $\beta$ changes. 


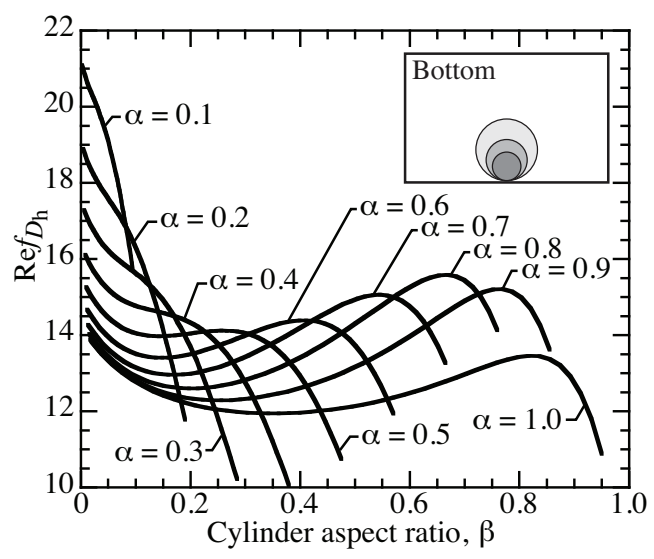

Figure 6: Reynolds-number-friction-factor product $\operatorname{Re} f_{\mathrm{D}_{\mathrm{h}}}$ as a function of the cylinderdiameter aspect ratio $\beta$ for ten values of the rectangular channel aspect ratio $\alpha$. The cylinder is placed on the middle of the floor of the rectangular channel. The shaded circles indicate how the cylinder diameter changes as $\beta$ changes. 


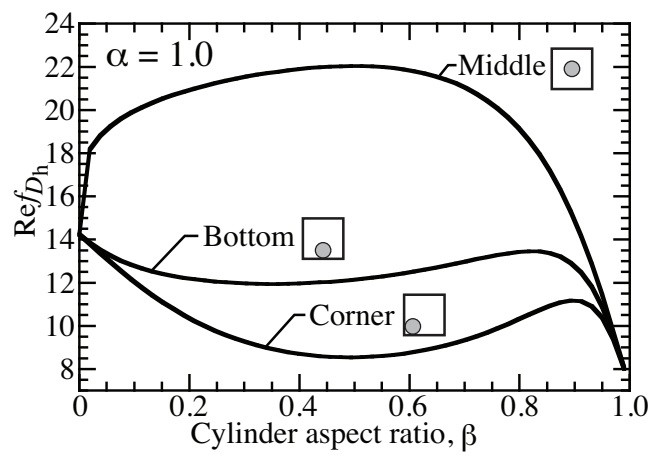

Figure 7: Reynolds-number-friction-factor product $\operatorname{Re} f_{\mathrm{D}_{\mathrm{h}}}$ as a function of the cylinderdiameter aspect ratio $\beta$ for rectangular-channel aspect ratio $\alpha=1.0$. The cylinder is placed either in the center of the channel, in the corner of the channel, or at the middle of the floor of the rectangular channel. 

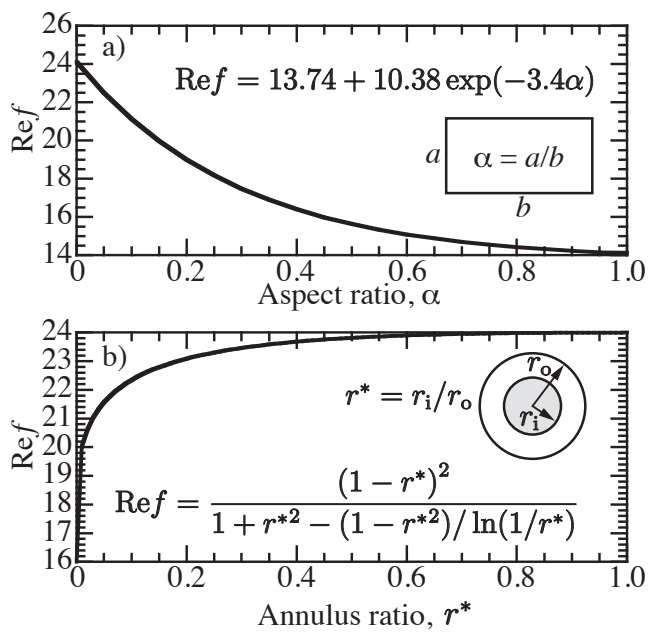

Figure 8: Well-known Ref correlations for fully developed flow in a) open rectangular channels and b) cylindrical annuli. In both cases, the Reynolds number is based on hydraulic diameter. 


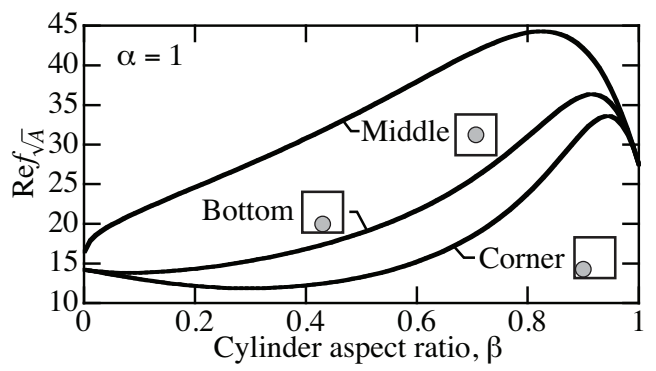

Figure 9: Reynolds-number-friction-factor product $\operatorname{Re} f_{\sqrt{A_{\mathrm{c}}}}$ as a function of the cylinderdiameter aspect ratio $\beta$ for rectangular-channel aspect ratio $\alpha=1.0$. The cylinder is placed either in the center of the channel, in the corner of the channel, or at the middle of the floor of the rectangular channel. 


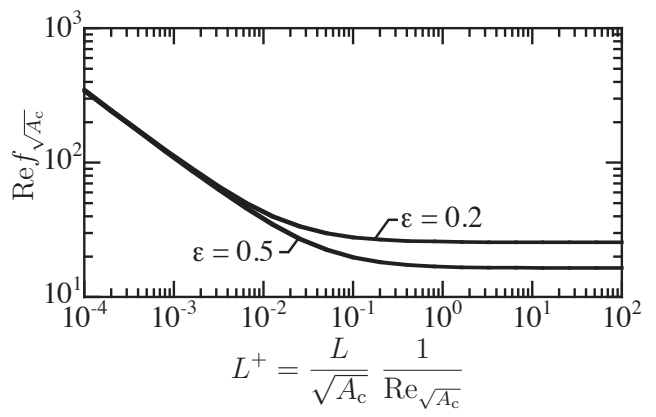

Figure 10: $\operatorname{Re} f$ as a function of $L^{+}$as predicted from Eq. 32 for two different aspect ratios. 


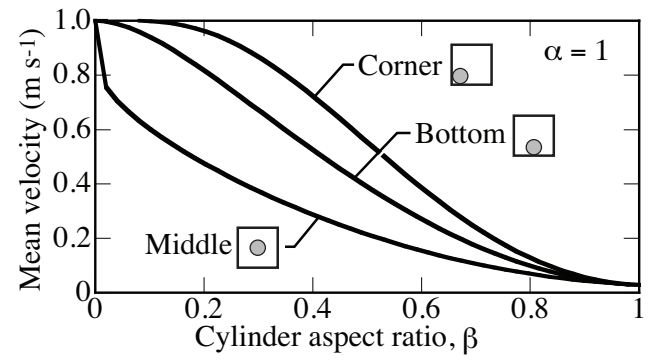

Figure 11: Mean inlet velocity as a function of cylinder aspect ratio $\beta$ for three probe positions. The pressure drop across a $20 \mathrm{~mm}$ channel length is fixed at $\Delta p=23 \mathrm{~Pa}$. The square channel is $1 \mathrm{~mm}$ on a side $(\alpha=1)$, and fluid properties of air at $900{ }^{\circ} \mathrm{C}$ are assumed. 


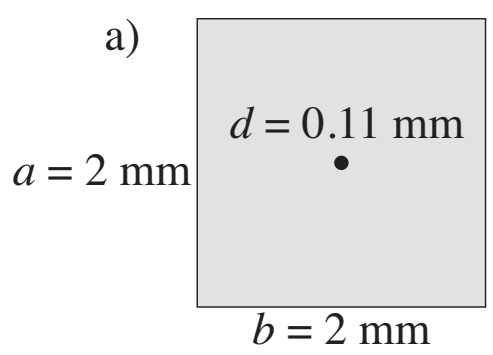

b)

$$
a=0.2 \mathrm{~mm} \stackrel{\bullet}{\square=2 \mathrm{~mm}}
$$

Figure 12: Two rectangular channels with a centered cylinder, both having $\beta \approx 0.055$. The upper channel has aspect ratio $\alpha=1$ and the lower channel has aspect ratio $\alpha=0.1$. 


\section{${ }_{600}$ List of Tables}

601

602

603

604

605

1 Coefficients for 7 th order polynomial fits for $\alpha=1,0.01<$ $\beta<1.0$, and three cylinder positions.

$\operatorname{Re} f_{D_{\mathrm{h}}}(\beta)=a_{0}+a_{1} \cdot \beta+a_{2} \cdot \beta^{2}+a_{3} \cdot \beta^{3}+a_{4} \cdot \beta^{4}+a_{5} \cdot \beta^{5}+$ $a_{6} \cdot \beta^{6}+a_{7} \cdot \beta^{7}$

43 
Table 1: Coefficients for 7th order polynomial fits for $\alpha=1,0.01<\beta<1.0$, and three cylinder positions.

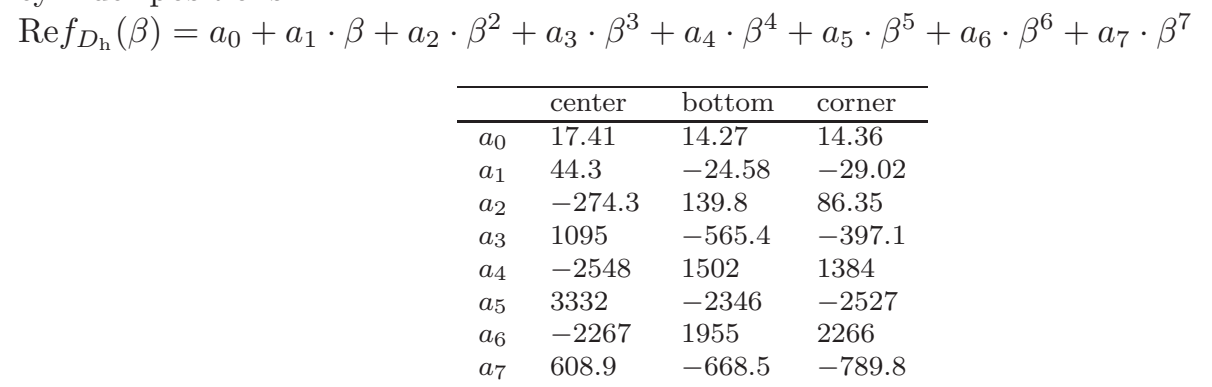

